\title{
Review Article \\ The Emerging Role of PEDF in Stem Cell Biology
}

\author{
Mina Elahy, Swati Baindur-Hudson, and Crispin R. Dass \\ Department of Biomedical and Health Sciences, Victoria University, St Albans, VIC 3021, Australia \\ Correspondence should be addressed to Crispin R. Dass, crispin.dass@vu.edu.au
}

Received 19 December 2011; Accepted 15 March 2012

Academic Editor: Susan E. Crawford

Copyright (C) 2012 Mina Elahy et al. This is an open access article distributed under the Creative Commons Attribution License, which permits unrestricted use, distribution, and reproduction in any medium, provided the original work is properly cited.

Encoded by a single gene, PEDF is a $50 \mathrm{kDa}$ glycoprotein that is highly conserved and is widely expressed among many tissues. Most secreted PEDF deposits within the extracellular matrix, with cell-type-specific functions. While traditionally PEDF is known as a strong antiangiogenic factor, more recently, as this paper highlights, PEDF has been linked with stem cell biology, and there is now accumulating evidence demonstrating the effects of PEDF in a variety of stem cells, mainly in supporting stem cell survival and maintaining multipotency.

\section{Introduction}

1.1. Introduction to PEDF. Pigment-epithelium-derived factor (PEDF) is a glycoprotein that belongs to the superfamily of serpin protease inhibitor proteins without inhibitory function, encoded by the gene SERPINF1 located on chromosome $17 \mathrm{p} 13$ which is well conserved in evolution [1]. It is a protein of 418 amino acids, with a size of $50 \mathrm{KDa}$ and widely expressed in most body tissues [2]. It is an extracellular protein which shows the secondary and tertiary structure of serpin and binds to collagen- 1 and heparin. The $\alpha \beta$-sheet is the dominant feature of the secondary structure and comprises the core structural domain of the protein, being closely involved in dynamic movements that are part of serpin function [3]. Existence of a reactive centre loop (RCL) is another feature of serpins, and it is a proteinase recognition site and a critical component of the function of serpins [4]. PEDF contains an RCL structure but the function of this is still unknown [4].

PEDF was originally isolated from the conditioned medium of cultured human fetal retinal pigment epithelium cells [4]. As mentioned before, it can be found in almost all tissues but the highest amount of expression has been observed in the eye, fetal and adult liver tissue, adult testis, ovaries, placenta, and the pancreas. A significant reduction in the expression of PEDF is found in senescent (aging) cells [5].

PEDF is a pluripotent molecule with neurotrophic qualities, and several biological activities have been ascribed to it, including antiangiogenic, antiproliferative, prodifferentiation, neuroprotective, anti-inflammatory, and antitumour properties [6-8]. The antiangiogenic properties and neurotrophic activities of the protein are due to peptides derived from the $\mathrm{N}$-terminal region of this protein, while the C-terminal peptides interact with its membrane receptor [7]. How PEDF controls all these biological processes and how it intercepts growth-promoting signals, accelerates cell death cascades, and prolongs cellular lifespan remains widely unknown but it presumably binds to cell surface receptors to trigger various signalling cascades. Evaluating the expression of different PEDF receptors (PEDF-Rs) could help to determine the specific biological responses of PEDF. Studies show that there are at least two different PEDFRs, specific to neural or endothelial cells, respectively [9]. The signalling pathways activated by PEDF regulate a number of key transcription factors including nuclear factor kappa-light-chain-enhancer of activated B cells (NF$\kappa \mathrm{B})[10]$, nuclear factor of activated T cells (NFATs) [11], peroxisome proliferator-activated receptor (PPAR- $\gamma$ ) [12], and the potent promigratory urokinase-type plasminogen activator (uPA)/receptor (uPAR) system [8, 13].

1.2. Introduction to Stem Cell Biology. Stem cells are recognised by two important features, firstly the self-renewal property and secondly the multilineage differentiation potential [14]. These characteristics make stem cells a preferred 
candidate for cell-based therapy for diseases such as neuronal damage [15].

Generally, stem cells are divided into two groups based on their origins: embryonic stem cells (ESCs) which are harvested from the inner cell mass of blastocysts and have the ability to produce all three embryonic germ layers-ectoderm, endoderm, and mesoderm and adult stem cells which are critical for tissue homeostasis. Adult stem cells support tissue regeneration and replacement of ongoing cell loss due to natural cell death and injury. These cells can be found in most tissues such as the brain, bone marrow, liver, and retina $[16,17]$.

Stem cells divide via mitosis and produce one daughter cell to replace the divided parent cell (and thereby maintain the stem cell pool) and one other cell which will go through the differentiation process. The cell which undergoes differentiation can do so to different types of cells. Recently, a phenomenon known as trans-differentiation has been identified which is when tissue-specific stem cells switch their lineage to that of some other tissue under suitable conditions. For example, bone marrow stem cells are able to transdifferentiate into skeletal muscle, smooth muscle, and neuronal cells [18].

In spite of the unique properties of stem cells (selfrenewal and differentiation), adult stem cells stay dormant through most of their lifetime and are activated under certain circumstances by specific environmental factors [19]. The specific surrounding environment includes stroma which contains fibroblasts, macrophages, neutrophils, endothelial cells and other differentiated cells, and the microenvironment around the stem cell [20].

The surrounding microenvironment of a stem cell is known as the stem cell niche and it includes different signalling areas which helps the daughter cell commit to different fates. Stem cells need to be held within the niche and this happens via adhesion between stem cells and underlying basement membrane or support cells. Upon division, if a cell is placed outside the niche, it commits to differentiation depending on the different microenvironmental stimuli and signalling it encounters in its new niche [19].

Stem cells have the capacity to react to a broad range of growth factors and signalling molecules and express many of the important downstream signal transduction components such as those signal transduction pathways that are present and may be active in stem cells. These include Notch, transforming growth factor beta (TGF $\beta$ ), Wnt, bone morphogenetic proteins (BMPs), Hedgehog, and Janus kinase/signal transducer and activator of transcription (Jak/Stat) family members [21].

\section{PEDF and Stem Cells}

2.1. PEDF and Human Embryonic Stem Cells. Human embryonic stem cells (hESCs) grown in vitro require feeder cells such as fibroblasts to maintain proliferation and pluripotency. In a study using microarray analysis to study the gene expression profile of human foreskin fibroblasts, a number of molecular targets were identified that are potentially involved in the ability of these cells to act as feeder cells for hESCs. The most likely target genes they found were PEDF, c-Kit, and leptin. These findings were supported by real-time polymerase chain reaction (RT-PCR) and virtual serial analysis of gene expression (SAGE) analysis. It has been hypothesized that the protein products of these genes may play an important role in the support of hESC growth by human foreskin fibroblasts [22].

Age-related macular degeneration (AMD) is one of the common causes of blindness in the elderly and is characterised by degeneration and loss of retinal pigmented epithelial (RPE) cells and photoreceptor cells in the macular region. Local expression of PEDF is significantly decreased in the eyes of patients with AMD [23]. There are two late forms of the disease: in geographic atrophy (also known as dry) there are confluent areas of RPE degeneration and loss in the macular region, while in neovascular AMD (also known as wet), there is growth of blood vessels from the choroid through Bruch's membrane to the subretinal spacea process known as choroidal neovascularization (CNV) $[24,25]$.

hESC-derived retinal pigment epithelium (hES-RPE) seems to be a promising strategy for cell replacement in this condition. Studies have shown that polarised hESRPE cells secrete high levels of PEDF and that culture medium containing this PEDF increased proliferation and supported the survival of retinal progenitor cells (RPCs) isolated from human fetal eyes [23]. This role of PEDF was confirmed by the addition of neutralising PEDF antibody to the culture media, which diminished or completely abolished the increase in RPC proliferation and survival.

Apart from the potential effects of PEDF on RPCs, PEDF also has at least three important effects on the health of RPEs. The first effect is the neuroprotective activity towards photoreceptors and other retinal neural cells against damage of cytotoxic injury. Second, its antiangiogenic effect prevents pathologic neovascularisation through inhibiting endothelial cell migration and promoting endothelial apoptosis. Third, its antiaging function inhibits premature senescence of both RPE cells and their neighbouring retinal cells [26] (Table 1).

\subsection{PEDF and Mesenchymal Stem Cells (MSCs). Bone} marrow-derived stem cells (BMSCs) are also called mesenchymal stem cells (MSCs) because these cells are able to differentiate into a variety of mesodermal tissues including bone, cartilage, and adipose [14]. One of the most abundant proteins identified in murine mesenchymal MSCconditioned medium is PEDF [27]. Immunofluorescent staining shows a high level of expression of PEDF in the rough endoplasmic reticulum/Golgi areas [27]. PEDF is also found to be located near the plasma membrane and in the extracellular space (considering the ability of PEDF to bind to collagen and proteoglycans in the extracellular matrix).

The role of bone-marrow-derived SCs (BMSCs) or MSCs in angiogenesis is not clear but it has been suggested that they may support and stabilise newly formed blood vessels. The process of angiogenesis is regulated by various factors that stimulate or inhibit angiogenesis. One of the key factors that 
TABLE 1: Effects of PEDF on different types of stem cells.

\begin{tabular}{llc}
\hline Stem cell type & PEDF effect & Reference \\
\hline Human embryonic stem cell & Proliferation, support, and survival & {$[22,23]$} \\
\hline \multirow{3}{*}{ Neural stem cell } & Self-renewal & {$[31-33]$} \\
& Maintenance of multipotency & \\
& Activation of cell division & \\
& Antiapoptotic effects & {$[35,37,38]$} \\
& Self-renewal & \\
Retinal stem cell & Cell expansion & Inhibits migration of and induces apoptosis of endothelial cells \\
& Regression of CNV (at low doses) & \\
& Development of neovascularisation (at high doses) & \\
\hline
\end{tabular}

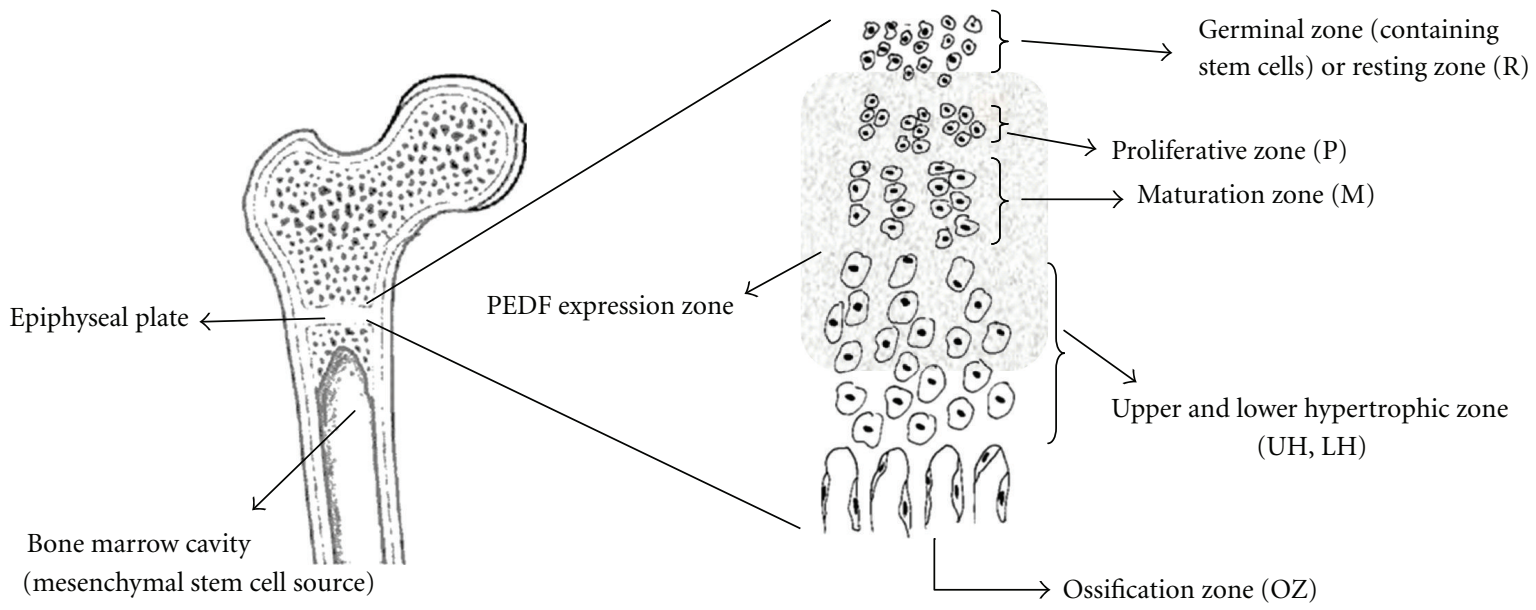

Figure 1

stimulates angiogenesis is vascular endothelial growth factor (VEGF). This factor is expressed in a wide range of cells including BMSCs. On the other hand, one of the key functions of the PEDF molecule is the inhibition of angiogenesis. In a study by Fan et al. (2011), it was observed that PEDF expression was much stronger than that of VEGF in BMSCs. Based on data acquired during that project, the authors concluded that BMSCs may not usually be an angiogenesispromoting population in a normal environment [28]. This view was supported by other findings that, in the presence of high cell numbers, MSCs are capable of inhibiting capillary growth [29].

During differentiation of MSCs to osteoblasts, expression of several genes begins and this includes PEDF. It has been shown that a high level of PEDF is expressed during early stages of bone development by osteoblasts and to a lesser extent in osteoclasts [30,31]. Osteoblasts and possibly osteoclasts are able to synthesise and release PEDF, and this protein has a critical role in normal and abnormal bone angiogenesis. In developing bones, blood vessel growth is localised. In locations such as the long bone growth plate, blood vessels selectively invade the region between hypertrophic chondrocytes and newly formed bone matrix
(Figure 1). These newly formed vessels allow migration of osteoblasts which leads to new bone matrix deposition and bone elongation. PEDF is expressed in the epiphyseal cartilage and in the areas of active bone remodelling in the primary spongiosa and periosteum of metaphyseal bone. There is a gradual decrease in the intensity of PEDF expression as chondrocytes differentiate toward the base of the growth plate $[30,32]$.

It has been found that PEDF secreted from enriched mouse bone marrow cells exhibiting surface markers characteristic of multipotent MSCs is able to attract fibroblasts and this property may play a role in regulating the cellular profile at the site of an injury. PEDF secretion happens in early postinjury stages rather than late postinjury and is due to the different cellular requirements during different stages. For example, PEDF released by resident stem cells may stimulate migration of cells needed early in regeneration, such as fibroblasts while inhibiting migration of cells such as endothelial cells needed for capillary formation at subsequent stages of healing [27].

2.3. PEDF and Neural Stem Cells (NSCs). In 2006 RamírezCastillejo et al. [33] showed that PEDF produced by the 
subventricular zone (SVZ) in the mouse brain promotes self-renewal and invigoration of slowly dividing adult neural stem cells (NSCs) in vitro [33]. It also stimulates the expansion of the stem cell niche in brain and induces differentiation toward the neuronal phenotype in multiple cell types [34]. In the subependymal zone (SEZ) of the adult mammalian brain, neuroblasts and oligodendrocytes are produced from NSCs through fast-dividing transitamplifying progenitors (TAPs). Vascular elements and several endothelium-derived factors are known to regulate the proliferation and/or survival of neural progenitors and PEDF is one of them. PEDF acts as an endogenous SEZ niche factor that can evoke NSC expansionary divisions [35].

Andreu-Agulló et al. (2009) found that PEDF could modulate the balance between symmetric and asymmetric divisions in NSCs. Essentially PEDF promoted self-renewing divisions and maintenance of a multipotent state in NSCs through its effects on Notch transcriptional activity. The mechanism by which this occurs appears to be complex. Activation of Notch receptors by membrane bound ligands results in the generation of an intracellular domain of Notch (NICD) which moves to the nucleus, where it binds the repressor $\mathrm{C}$ promoter-binding factor 1 (CBF1). $\mathrm{CBF} 1$ can then bind to the promoters and initiate transcription of genes in the Hes1 and Herp families and, possibly, the Egfr gene [35]. This results in the effects of Notch signalling, that is, increase in self-renewal of NSCs.

So what is the role of PEDF in all this? Notch activity is attenuated by the interplay between transcriptional coactivators and corepressors. One of these is $\mathrm{N}-\mathrm{CoR}$, which is a corepressor for a number of nuclear receptors and can bind to CBF1. It was postulated that the CBF1-binding sites in the Hes1 and Egfr promoters were occupied by N-CoR, repressing the transcription of these genes (Figure 2(a)). It was found that, in the presence of PEDF, N-CoR moves from a nuclear to cytoplasmic location and that PEDF removes $\mathrm{N}$-CoR (the repressor) from the CBF1-binding sites in the promoters of the Hes1 and Efgr genes, thus allowing CBF1 (the inducer) to bind and activate these genes (Figure 2(b)). Further experiments suggested that PEDF carries out these activities through a noncanonical activation of the NF- $\kappa \mathrm{B}$ pathway [35].

2.4. Stem Cells Overexpressing the PEDF Gene. MSCs have been shown to differentiate into endothelial cells (ECs) and vascular smooth muscle cells (VSMCs) and incorporate into the new blood vessel wall and form vascular tubes. MSCs have an explicit role in various proangiogenic models although, interestingly, they have an antiangiogenic effect on corneal wound healing after chemical injury [36]. Excessive vascularisation can lead to pathological situations. Studies show that MSCs contribute to the formation of choroidal neovascularisation $(\mathrm{CNV})$ and they can differentiate into both vascular and extravascular cells in CNV [37]. CNV is a major form of ocular angiogenesis which leads to visual loss.

The mechanism of CNV is complex but, basically, a disrupted balance between angiogenic and antiangiogenic factors like VEGF as an angiogenic stimulator and PEDF as an

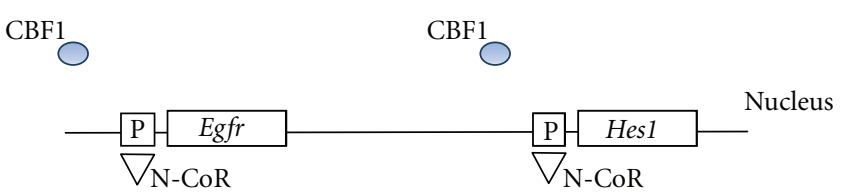

(a)

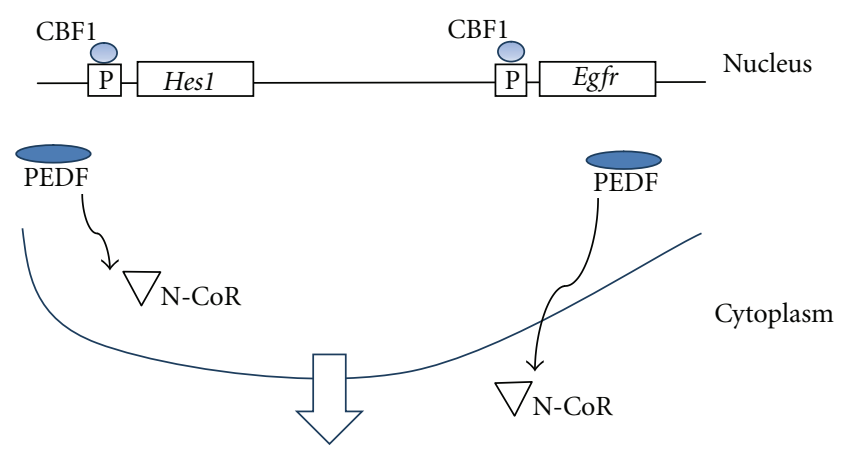

(b)

Figure 2

inhibitor of angiogenesis is the cause. It has been suggested that PEDF may cause regression of CNV promoted by VEGF [38]. The effects of PEDF on CNV are complicated: while PEDF suppresses the growth of endothelial cells exposed to fibroblast growth factor-2 (FGF2), it evokes proliferation of endothelial cells in the presence of high VEGF levels. In another report, PEDF showed concentration-dependent effects on CNV and endothelial cell function: low doses are inhibitory, but high doses can increase neovascularisation [39].

In a study by Hou et al. (2010), it was observed that injecting MSCs transduced with adenoviral vectors expressing PEDF (AdPEDF) caused a regression in neovascularisation as a result of the CNVs being encapsulated in retinal pigment epithelial cells (RPEs) [37].

Deterioration of photoreceptor cells may be caused by a continuous degeneration of retinal pigment epithelium (RPE) cells and can lead to vision loss. Adenovirally transduced bone marrow stromal (stem) cells carrying the PEDF gene exhibited the typical morphologies of RPE cells in vitro. Furthermore, when these cells were injected into the superior subretinal space of Royal College of Surgeons (RCS) rats, a well-established model of retinal degeneration due to defective phagocytosis of photoreceptor outer segments by the RPE, these cells produced more pronounced rescue effects than nontransduced cells. This suggests that PEDF has potency to protect photoreceptor cells from degeneration [40].

Stem cell therapy, especially using MSCs, has been considered as a promising method for treating myocardial infarction (MI) [41]. In a study where myocardial infarction was induced in C57BL/6 mice, it had been observed that MSCs could ameliorate MI injury, but that MSCs derived from older donors had less efficacy. It was found that older 
MSCs secreted higher levels of PEDF than younger ones. Furthermore, infarcts treated with (AdPEDF-) transduced MSCs that over-expressed PEDF contained fewer ECs, VSMCs, and macrophages but had increased number of fibroblasts [42]. They concluded that it was the increased expression of PEDF in aged MSCs that impaired their therapeutic efficacy.

\section{Conclusion}

PEDF has a range of functions in different tissues and cells; however, the impact of this interesting protein on stem cells is not yet clear. PEDF expressed in feeder cells appears to have a supportive effect on stem cells such as hESCs and RPCs. PEDF affects NSCs via the Notch transcriptional pathway, promoting self-renewing divisions, maintaining a multipotent state of these cells and supporting expansion of the stem cell niche. Stem cells transduced with adenoviral vectors carrying PEDF exhibit potential for enhanced stem cell therapy in diseases such as macular degeneration. However, the effects of PEDF on stem cells in other disease states are clear. Whilst PEDF generally causes regression of CNV established by VEGF, the effects may be concentration dependent-both on the concentration of VEGF and PEDF. As for myocardial infarction, the presence of excessive PEDF is actually reported to reduce the efficacy of stem cell therapy. To conclude, the role of PEDF in stem cells in various pathologies does differ, and while counterproductive in some cases such as myocardial infarction, it may have a positive role to play in some instances. Further studies on PEDF and stem cells will help illuminate such instances and whether they can be used therapeutically.

\section{References}

[1] Z. Xu, S. Fang, Y. Zuo et al., "Combination of pigment epithelium-derived factor with radiotherapy enhances the antitumor effects on nasopharyngeal carcinoma by downregulating vascular endothelial growth factor expression and angiogenesis," Cancer Science, vol. 102, no. 10, pp. 1789-1798, 2011.

[2] Q. Gong, X. Yang, W. Cai, G. Gao, and Z. Yang, "Expression and purification of functional epitope of pigment epitheliumderived factor in E. coli with inhibiting effect on endothelial cells," Protein Journal, vol. 29, no. 3, pp. 167-173, 2010.

[3] S. T. Olson and P. G. Gettins, "Regulation of proteases by protein inhibitors of the serpin superfamily," Progress in Molecular Biology and Translational Science C, vol. 99, pp. 185240, 2011.

[4] J. Tombran-Tink, S. Aparicio, X. Xu et al., "PEDF and the serpins: phylogeny, sequence conservation, and functional domains," Journal of Structural Biology, vol. 151, no. 2, pp. 130-150, 2005.

[5] T. Samkharadze, M. Erkan, C. Reiser-Erkan et al., "Pigment epithelium-derived factor associates with neuropathy and fibrosis in pancreatic cancer," American Journal of Gastroenterology, vol. 106, no. 5, pp. 968-980, 2011.

[6] T. Akiyama, C. R. Dass, Y. Shinoda, H. Kawano, S. Tanaka, and P. F. M. Choong, "PEDF regulates osteoclasts via osteoprotegerin and RANKL," Biochemical and Biophysical Research Communications, vol. 391, no. 1, pp. 789-794, 2010.
[7] F. Sánchez-Sánchez, J. D. Aroca-Aguilar, I. Segura et al., "Expression and purification of functional recombinant human pigment epithelium-derived factor (PEDF) secreted by the yeast Pichia pastoris," Journal of Biotechnology, vol. 134, no. 1-2, pp. 193-201, 2008.

[8] M. L. Broadhead, C. R. Dass, and P. F. Choong, "In vitro and in vivo biological activity of PEDF against a range of tumors," Expert Opinion on Therapeutic Targets, vol. 13, no. 12, pp. 1429-1438, 2009.

[9] J. L. Orgaz, O. Ladhani, K. S. Hoek et al., "Loss of pigment epithelium-derived factor enables migration, invasion and metastatic spread of human melanoma," Oncogene, vol. 28, no. 47, pp. 4147-4161, 2009.

[10] T. Yabe, D. Wilson, and J. P. Schwartz, "NF $\kappa$ B activation is required for the neuroprotective effects of pigment epithelium-derived Factor (PEDF) on cerebellar granule neurons," The Journal of Biological Chemistry, vol. 276, no. 46, pp. 43313-43319, 2001.

[11] T. A. Zaichuk, E. H. Shroff, R. Emmanuel, S. Filleur, T. Nelius, and O. V. Volpert, "Nuclear factor of activated T cells balances angiogenesis activation and inhibition," Journal of Experimental Medicine, vol. 199, no. 11, pp. 1513-1522, 2004.

[12] T. C. Ho, S. L. Chen, Y. C. Yang, C. L. Liao, H. C. Cheng, and Y. P. Tsao, "PEDF induces p53-mediated apoptosis through PPAR gamma signaling in human umbilical vein endothelial cells," Cardiovascular Research, vol. 76, no. 2, pp. 213-223, 2007.

[13] C. R. Dass and P. F. M. Choong, "uPAR mediates anticancer activity of PEDF," Cancer Biology and Therapy, vol. 7, no. 8, pp. 1262-1270, 2008.

[14] Y. L. Si, Y. L. Zhao, H. J. Hao, X. B. Fu, and W. D. Han, "MSCs: biological characteristics, clinical applications and their outstanding concerns," Ageing Research Reviews, vol. 10, no. 1, pp. 93-103, 2011.

[15] C. Trento and F. Dazzi, "Mesenchymal stem cells and innate tolerance: biology and clinical applications.", Swiss Medical Weekly, vol. 140, article w13121, 2010.

[16] A. G. Smith, "Embryo-derived stem cells: of mice and men," Annual Review of Cell and Developmental Biology, vol. 17, pp. 435-462, 2001.

[17] L. Li and T. Xie, "Stem cell niche: structure and function," Annual Review of Cell and Developmental Biology, vol. 21, pp. 605-631, 2005.

[18] R. M. Backly and R. Cancedda, "Bone marrow stem cells in clinical application: harnessing paracrine roles and niche mechanisms," Advances in Biochemical Engineering/Biotechnology, vol. 123, pp. 265-292, 2010.

[19] A. Wilson, E. Laurenti, G. Oser et al., "Hematopoietic stem cells reversibly switch from dormancy to self-renewal during homeostasis and repair," Cell, vol. 135, no. 6, pp. 1118-1129, 2008.

[20] T. Yin and L. Li, “The stem cell niches in bone," The Journal of Clinical Investigation, vol. 116, no. 5, pp. 1195-1201, 2006.

[21] T. Reya, S. J. Morrison, M. F. Clarke, and I. L. Weissman, "Stem cells, cancer, and cancer stem cells," Nature, vol. 414, no. 6859, pp. 105-111, 2001.

[22] S. V. Anisimov, N. S. Christophersen, A. S. Correia et al., "Identification of molecules derived from human fibroblast feeder cells that support the proliferation of human embryonic stem cells," Cellular and Molecular Biology Letters, vol. 16, no. 1, pp. 79-88, 2011. 
[23] D. Zhu, X. Deng, C. Spee et al., "Polarized secretion of PEDF from human embryonic stem cell-derived RPE promotes retinal progenitor cell survival," Investigative Ophthalmology and Visual Science, vol. 52, no. 3, pp. 1573-1585, 2011.

[24] J. D. Weiland, A. K. Cho, and M. S. Humayun, "Retinal prostheses: current clinical results and future needs," Ophthalmology, vol. 118, no. 11, pp. 2227-2237, 2010.

[25] P. van Wijngaarden and S. H. Qureshi, "Inhibitors of vascular endothelial growth factor (VEGF) in the management of neovascular age-related macular degeneration: a review of current practice," Clinical \& Experimental Optometry, vol. 91, no. 5, pp. 427-437, 2008.

[26] I. H. Pang, H. Zeng, D. L. Fleenor, and A. F. Clark, "Pigment epithelium-derived factor protects retinal ganglion cells," BMC Neuroscience, vol. 8, article 11, 2007.

[27] H. Sarojini, R. Estrada, H. Lu et al., "PEDF from mouse mesenchymal stem cell secretome attracts fibroblasts," Journal of Cellular Biochemistry, vol. 104, no. 5, pp. 1793-1802, 2008.

[28] W. Fan, R. Crawford, and Y. Xiao, "The ratio of VEGF/PEDF expression in bone marrow mesenchymal stem cells regulates neovascularization," Differentiation, vol. 81, no. 3, pp. 181191, 2011.

[29] K. Otsu, S. Das, S. D. Houser, S. K. Quadri, S. Bhattacharya, and J. Bhattacharya, "Concentration-dependent inhibition of angiogenesis by mesenchymal stem cells," Blood, vol. 113, no. 18, pp. 4197-4205, 2009.

[30] J. Tombran-Tink and C. J. Barnstable, "Osteoblasts and osteoclasts express PEDF, VEGF-A isoforms, and VEGF receptors: Possible mediators of angiogenesis and matrix remodeling in the bone," Biochemical and Biophysical Research Communications, vol. 316, no. 2, pp. 573-579, 2004.

[31] C. Chiellini, O. Cochet, L. Negroni et al., "Characterization of human mesenchymal stem cell secretome at early steps of adipocyte and osteoblast differentiation," BMC Molecular Biology, vol. 9, p. 26, 2008.

[32] G. M. Y. Quan, J. Ojaimi, Y. Li, V. Kartsogiannis, H. Zhou, and P. F. M. Choong, "Localization of pigment epithelium-derived factor in growing mouse bone," Calcified Tissue International, vol. 76, no. 2, pp. 146-153, 2005.

[33] C. Ramírez-Castillejo, F. Sánchez-Sánchez, C. Andreu-Agulló et al., "Pigment epithelium-derived factor is a niche signal for neural stem cell renewal," Nature Neuroscience, vol. 9, no. 3, pp. 331-339, 2006.

[34] Y. Mirochnik, A. Aurora, F. T. Schulze-Hoepfner et al., "Short pigment epithelial-derived factor-derived peptide inhibits angiogenesis and tumor growth," Clinical Cancer Research, vol. 15, no. 5, pp. 1655-1663, 2009.

[35] C. Andreu-Agulló, J. M. Morante-Redolat, A. C. Delgado, and I. Fariñas, "Vascular niche factor PEDF modulates notchdependent stemness in the adult subependymal zone," Nature Neuroscience, vol. 12, no. 12, pp. 1514-1523, 2009.

[36] Y. O. Joo, K. K. Mee, S. S. Mi et al., "The anti-inflammatory and anti-angiogenic role of mesenchymal stem cells in corneal wound healing following chemical injury," Stem Cells, vol. 26, no. 4, pp. 1047-1055, 2008.

[37] H. Y. Hou, H. L. Liang, Y. S. Wang et al., "A therapeutic strategy for choroidal neovascularization based on recruitment of mesenchymal stem cells to the sites of lesions," Molecular Therapy, vol. 18, no. 10, pp. 1837-1845, 2010.

[38] J. P. Tong and Y. F. Yao, "Contribution of VEGF and PEDF to choroidal angiogenesis: a need for balanced expressions," Clinical Biochemistry, vol. 39, no. 3, pp. 267-276, 2006.
[39] R. S. Apte, R. A. Barreiro, E. Duh, O. Volpert, and T. A. Ferguson, "Stimulation of neovascularization by the antiangiogenic factor PEDF," Investigative Ophthalmology and Visual Science, vol. 45, no. 12, pp. 4491-4497, 2004.

[40] S. Arnhold, P. Heiduschka, H. Klein et al., "Adenovirally transduced bone marrow stromal cells differentiate into pigment epithelial cells and induce rescue effects in RCS rats," Investigative Ophthalmology and Visual Science, vol. 47, no. 9, pp. 4121-4129, 2006.

[41] R. Passier, L. W. van Laake, and C. L. Mummery, "Stem-cellbased therapy and lessons from the heart," Nature, vol. 453, no. 7193, pp. 322-329, 2008.

[42] H. Liang, H. Hou, and W. Yi, "Increased expression of pigment epithelium-derivedfactor in aged mesenchymal stem cells impairs their therapeutic efficacy for attenuating myocardial infarction injury," European Heart Journal. In press. 

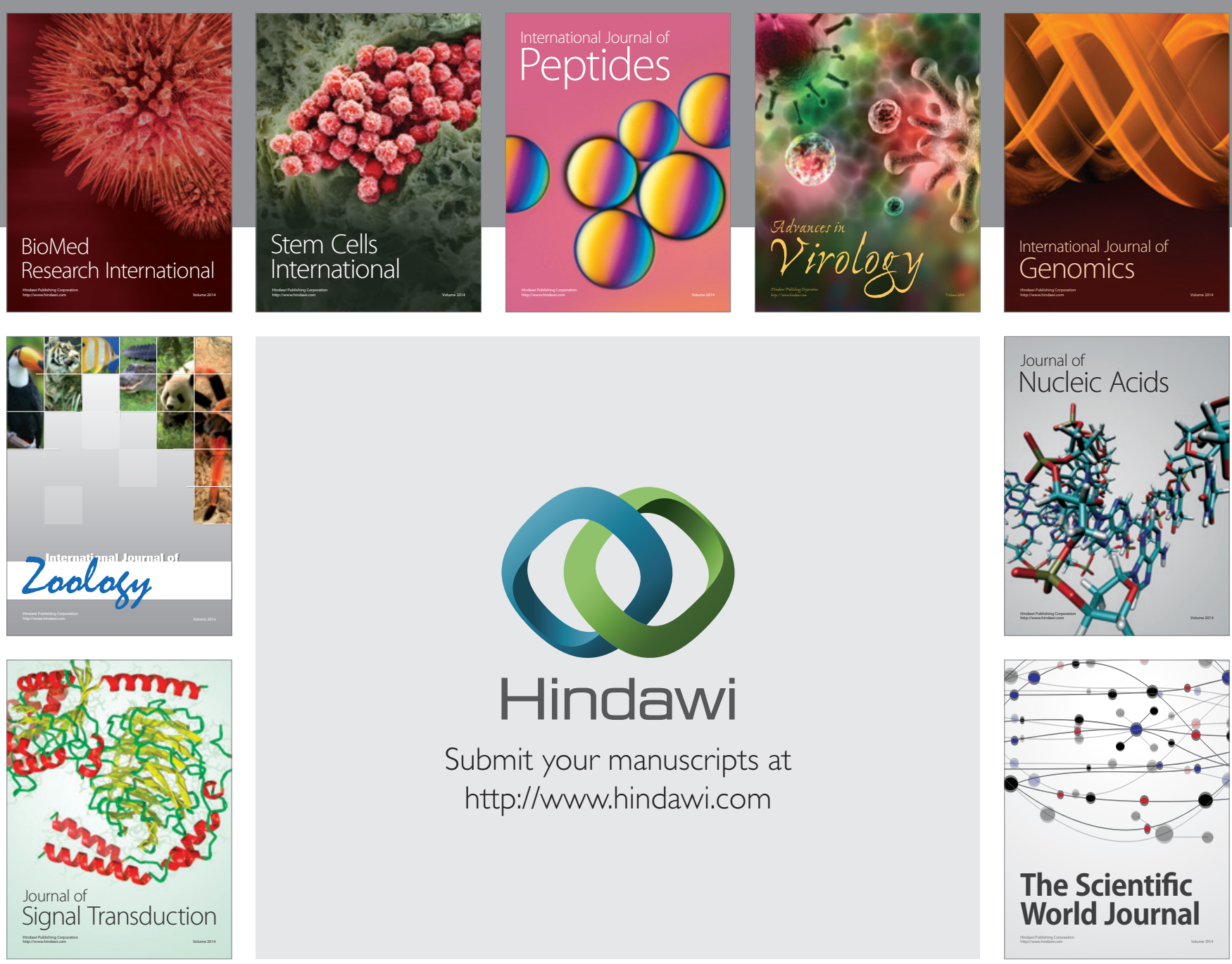

Submit your manuscripts at

http://www.hindawi.com
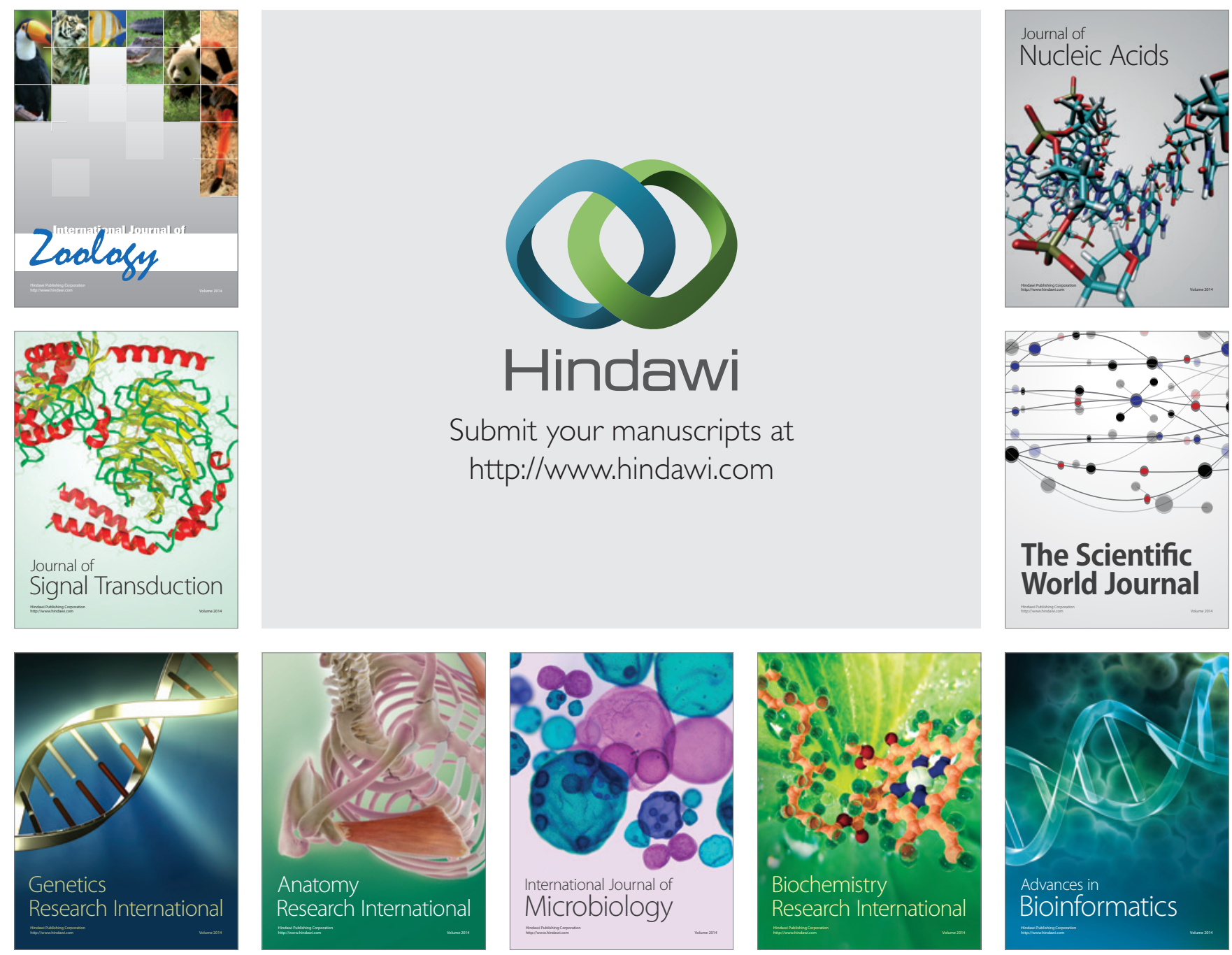

The Scientific World Journal
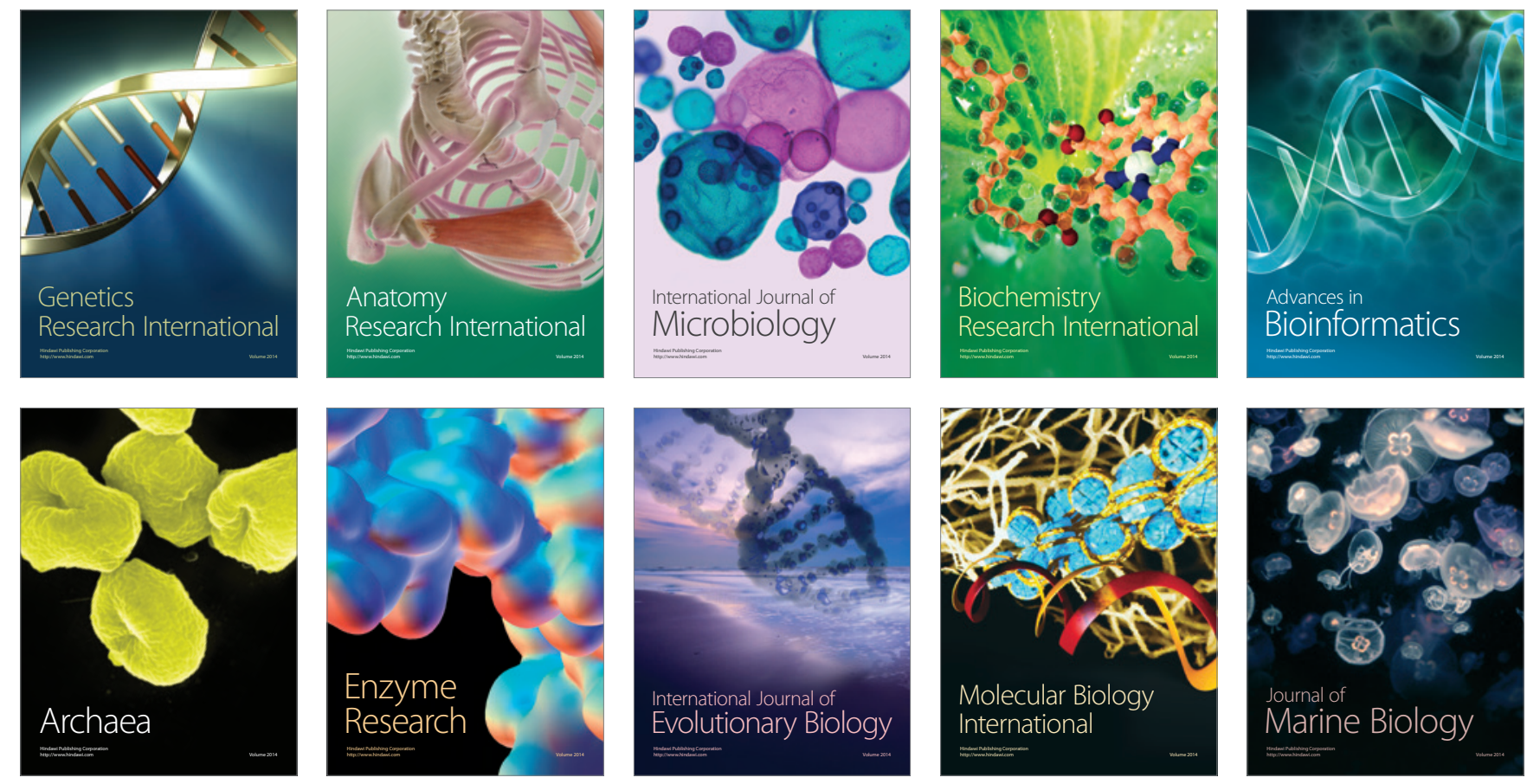